\title{
An atomic force microscope integrated with a helium ion microscope for correlative nanoscale characterization
}

\author{
Santiago H. Andany ${ }^{1}$, Gregor Hlawacek ${ }^{2}$, Stefan Hummel ${ }^{3}$, Charlène Brillard ${ }^{1}$, \\ Mustafa Kangül ${ }^{1}$ and Georg E. Fantner ${ }^{* 1}$
}

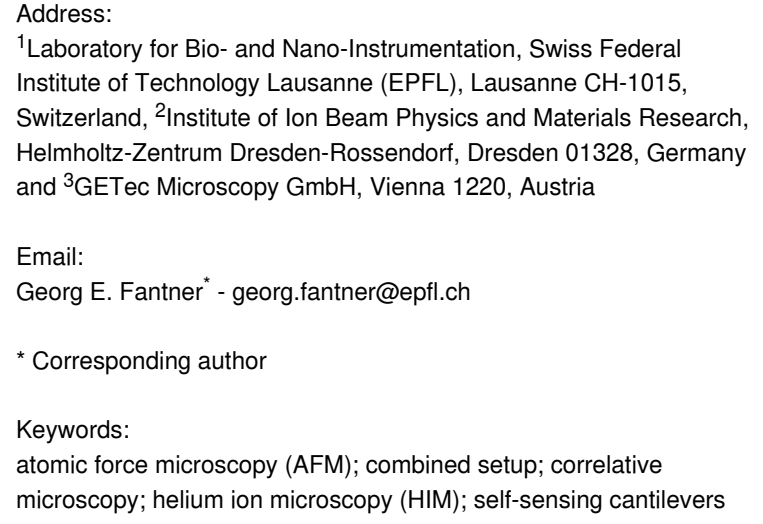

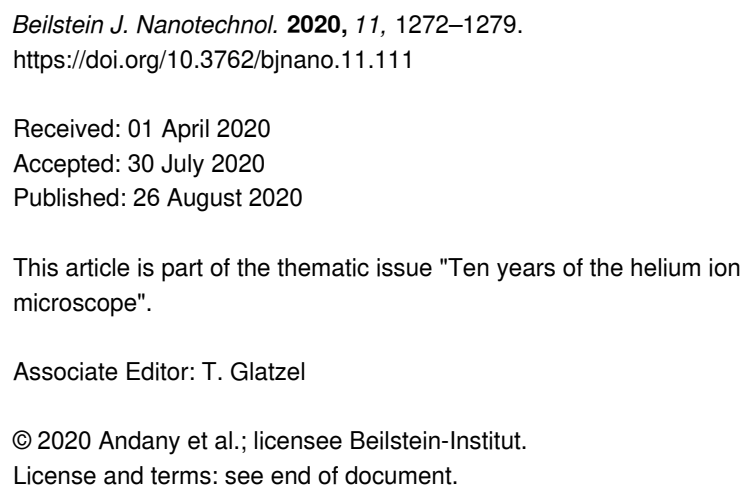

\begin{abstract}
In this work, we report on the integration of an atomic force microscope (AFM) into a helium ion microscope (HIM). The HIM is a powerful instrument, capable of imaging and machining of nanoscale structures with sub-nanometer resolution, while the AFM is a well-established versatile tool for multiparametric nanoscale characterization. Combining the two techniques opens the way for unprecedented in situ correlative analysis at the nanoscale. Nanomachining and analysis can be performed without contamination of the sample and environmental changes between processing steps. The practicality of the resulting tool lies in the complementarity of the two techniques. The AFM offers not only true 3D topography maps, something the HIM can only provide in an indirect way, but also allows for nanomechanical property mapping, as well as for electrical and magnetic characterization of the sample after focused ion beam materials modification with the HIM. The experimental setup is described and evaluated through a series of correlative experiments, demonstrating the feasibility of the integration.
\end{abstract}

\section{Introduction}

Shortly after the invention of the atomic force microscope (AFM) in 1986 [1], efforts were made towards combining this scanning probe microscopy technique with electron beam and ion beam techniques for correlative nanoscale characterization and nanoscale fabrication. The motivation was driven by the new opportunity to investigate and transform features in situ with complementary techniques, thus revealing maximum information without breaking the vacuum. The scanning electron microscope (SEM) was first combined with scanning tunneling microscopy (STM) [2,3], allowing for the visual observation at the tip-sample interaction point with the SEM. Later, Ermakov et al. [4] successfully integrated an AFM into an SEM for the 
first time, enabling correlative imaging on electrically insulating samples. In this first attempt, the readout of cantilever deflection was achieved using the electron beam itself. Shortly after, better performing combined setups were described utilizing more conventional self-sensing [5] and optical [6] techniques for the readout of cantilever deflection. Since then, more advanced and versatile combined instruments have been proposed for a broad spectrum of applications in nanoscale characterization and nanoscale fabrication inside SEM and focused ion beam (FIB) setups [7-11].

Given the extent of the interest sparked by SEM/FIB-AFM systems, it is reasonable to assume that the most recent ion beam microscope, the helium ion microscope (HIM), would be a serious contender for the use in combined setups in conjunction with AFM. Introduced by Ward et al. [12], the imaging capability of the HIM surpasses that of the SEM in terms of lateral resolution, depth of field, surface sensitivity, and ability to image electrically insulating samples [13]. Furthermore, nanoscale structuration with noble gas ions can yield sub-10 nm structures without unwanted metal ion implantation, a sizeable advantage over traditional gallium-ion FIBs. The resulting combined AFM-HIM instrument would, therefore, profit from the sub-nanometer lateral resolution of the HIM and the atomic resolution in the vertical axis of the AFM, proving particularly powerful for high-resolution correlative characterization of nonconductive samples.

With the integrated electron flood gun (FG) of the HIM providing charge neutralization, uncoated polymers and biological samples can be imaged with high resolution while the AFM would bring complementary information such as laterally resolved mechanical properties. These multiparametric measurements have previously been difficult to obtain as sample preparation of such samples for SEM or TEM are often incompatible with the needs of high-resolution AFM measurements.

AFM is also useful in assisting helium ion beam lithography. Many resists, including poly(methyl methacrylate) (PMMA), have higher sensitivities to helium ion irradiation than to electron irradiation in terms of charge per area [14]. Patterning resolution down to $4 \mathrm{~nm}$ has been demonstrated on HSQ resist [15], surpassing electron beam lithography, which greatly suffers from the proximity effect. In a combined AFM-HIM setup, the AFM could be used, in situ, in between exposures to assess the shrinkage, stiffness change or sputtering of the resist. More applications such as conductive AFM, piezo-force microscopy or magnetic force microscopy are within reach of the presented technology and would make AFM-HIM appealing to the microelectronics and materials research community.

\section{Instrumentation}

Spatial constraints inside SEMs and ion microscopes often dictate the feasibility of the integration of the AFM. Compact AFM setups have to fit around the host microscope as not to hinder excessively its capabilities. The reported AFM integration is depicted in Figure 1. The prototype tip-scanning AFM scan head is designed explicitly for correlative analysis inside electron and ion-beam microscopes. Unlike sample scanning
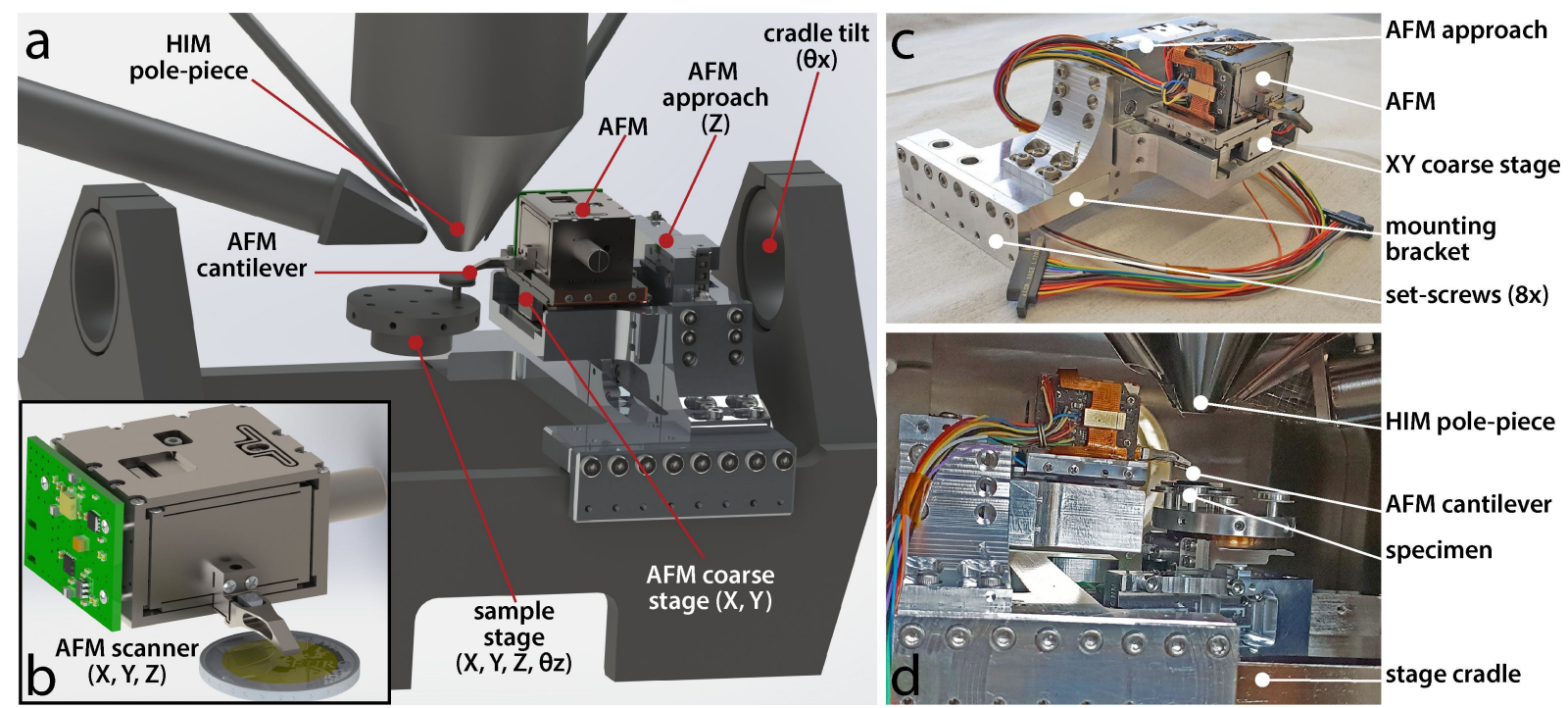

Figure 1: AFM assembly and integration inside a Zeiss ORION NanoFab helium ion microscope. a) Simplified CAD rendering of the AFM assembly mounted onto the HIM cradle and b) detailed view of the AFM scan head with a 2 EUR coin for scale. c) Annotated photograph of the AFM assembly and d) after being mounted inside the chamber of the HIM. 
solutions $[9,16]$, where the sample is raster-scanned relative to a stationary cantilever, a tip-scanning configuration $[10,17]$ requires no alteration of the sample stage and has the advantage of having the sample stationary within the field of view of the HIM during AFM imaging. The scanner is a flexure design with serial kinematics [18] and the cantilever is mounted at the end of a low-profile protruding $z$-flexure, which fits seamlessly between the pole piece and the sample. The assembly is made from grade-5 titanium (Ti-6Al-4V) and the three axes of motion are actuated by stack-piezo actuators, offering an achievable scan range of $30 \times 30 \times 12 \mu \mathrm{m}$.

The reported AFM uses a self-sensing readout for measuring cantilever deflection [19]. Although optical beam deflection (OBD) techniques are traditionally preferred for their lower noise, they require bulky components and precise optical alignment. Prohibitively large instruments with optical readout are difficult to integrate into electron or ion microscopes and re-alignment of the optics is very impractical, as it requires the user to vent the chamber. Using self-sensing readout addresses these concerns $[5,10,17]$ and also significantly improves the usability of the correlative instrument for users outside of the AFM community. The reported AFM uses silicon cantilever probes with boron-doped $1 \mathrm{k} \Omega$ sensing elements, connected in a Wheatstone bridge configuration with aluminum tracks [20,21] and with single crystal diamond (SCD) tips that are commercially available (SCL-SensorTech Fabrication GMBH, Vienna, Austria). The SCD tips (tip radius below $15 \mathrm{~nm}$, height $12-16 \mu \mathrm{m})$, which are glued to the cantilevers, offer high aspect ratio and excellent resistance to wear [22]. Because tip wear is the main cause of cantilever failure, and because cantilever exchange in the HIM requires the user to vent the chamber, wear resistance is critically important.

To maneuver the AFM relative to the sample and the ion beam, the AFM is mounted onto a coarse stage consisting of a custombuilt $X Y$ stick-slip positioner, which in turn is attached to a vertical approach mechanism built around a linear, stick-slip piezo actuator (Picomotor ${ }^{\mathrm{TM}}$ 8301-UHV, Newport Corporation, CA, USA). The AFM assembly tilts together with the sample stage. The three orthogonal translational degrees of freedom of the sample are decoupled from the AFM coarse positioning stage, as shown in Figure 1a. The integration of the AFM into the HIM requires no alteration of the HIM microscope stage. The AFM assembly is positioned onto the HIM cradle and secured with set screws pressing firmly on the sides of the cradle. Electrical connections necessary for AFM operation are cabled through a CF40 flange. After opening the microscope door, the AFM head can be removed seamlessly from the chamber for cantilever exchange thanks to a spring-loaded kinematic mount.
Operating the AFM inside the HIM chamber has drawbacks regarding resolution and noise performance. Ideally, the mechanical loop between the cantilever probe and the sample needs to be compact and stiff for optimal AFM performance. The mechanical loop for the reported tip-scanning AFM includes the AFM scan head, coarse stage and stationary sample stage. However, after integration with the HIM, stiffness is degraded through the incorporation of the HIM cradle, the fouraxis sample stage, and the mounting bracket to the mechanical loop. This reduces rigidity and makes the AFM more susceptible to vibrations present inside of the HIM chamber.

The AFM and motorized coarse stages are controlled with a custom-made AFM software [23], a standalone FPGA (USB7856R OEM, National Instruments, Austin TX, USA), a highvoltage piezo amplifier (Techproject, Vienna, Austria) and a stick-slip controller (8742-4 PicomotorTM drive, Newport Corporation). The AFM can operate in contact mode and in an off-resonance mode based on force-distance curves [24]. In this off-resonance mode, which we refer to as off-resonance tapping (ORT), the cantilever is moved sinusoidally up and down the sample using the $z$-piezo of the scanner, causing intermittent contact between the cantilever and the sample [25]. The maximum interaction force is computed and used as feedback by the controller, providing fine force control, reducing shear forces and thus preserving the tip and the sample [26,27]. Operating in ORT mode instead of amplitude-modulation AFM (AM-AFM) is particularly advantageous in vacuum, where the Q-factor of the cantilever is about one order of magnitude higher than in air. Indeed, AM-AFM imaging bandwidth is inversely proportional to the Q-factor [28] and is therefore very slow in vacuum [29]. In ORT, the tapping rate is a least one order of magnitude below the first resonance of the cantilever and hence the enhancement of the Q-factor in vacuum is less detrimental to the achievable scan speed.

\section{Results}

The system has been experimentally tested on a variety of sample surfaces in contact and off-resonance imaging modes, demonstrating the feasibility of the integration through a series of three experiments. Correlative AFM and HIM imaging is demonstrated in Figure 2 by imaging silicon nanopillars [30]. The HIM offers a large field of view, which allows for the cantilever to be navigated onto the region of interest (Figure 2b,c) to perform AFM topography imaging (Figure 2d).

PMMA has traditionally been used as a positive resist in electron beam lithography. Helium ion beam lithography has emerged as a powerful technique to achieve even smaller feature size thanks to higher resist sensitivity, reduced proximity effect and small spot size [15]. Upon ion beam exposure, chain 

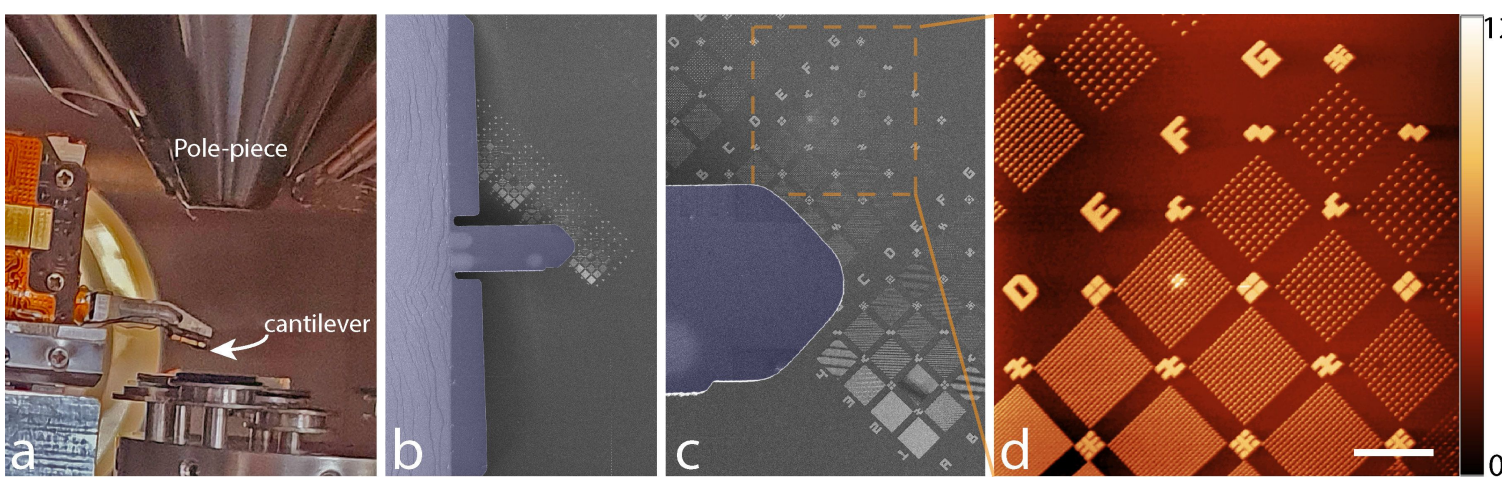

$120 \mathrm{~nm}$

Figure 2: Correlative imaging in process on silicon pillars. a) Optical image showing how the AFM cantilever is positioned at the end of a low-profile, overhanging structure, which fits between the pole piece and the sample. b, c) The cantilever (colorized in purple on the HIM images) can be navigated by making use of the large FOV image provided by the HIM. d) AFM height image of Si nanopillars taken in off-resonance tapping mode. Scale bar $5 \mu \mathrm{m}$.

scission occurs leaving the exposed region soluble in a suitable developer. Very high ion doses also break short side chains that later cross-link, allowing PMMA to be also used as a negative resist [31]. Chain scission leads to volume loss through the release of gas molecules, and this leads to the shrinkage of exposed PMMA [32], which can be easily quantified using AFM. In a second experiment, we tested the effect on a PMMA thin film exposed to different doses under the focused He ion beam. Figure $3 \mathrm{a}$ and Figure $3 \mathrm{~b}$ show two AFM topography images of PMMA exposed to a dose of $1 \times 10^{13} \mathrm{~cm}^{-2}$ and $3 \times 10^{13} \mathrm{~cm}^{-2} 30 \mathrm{keV}$ He ions, respectively, as well as the corresponding height profiles of the irradiated PMMA surface.

Focused ion beam damage and implantation can hinder the imaging and nanoscale fabrication capabilities of the HIM [33] and studying these local defects created at the micro- and nano- scale can provide valuable information towards understanding these limitations. For example, a focused helium ion beam can locally destroy the crystalline structure of silicon and lead to the growth of amorphous silicon bubbles at the surface [34]. Furthermore, focused helium ion beam exposure inside a HIM can be used as a way of locally replicating the harsh radiation conditions found in nuclear fission and fusion reactors, to study the response of structural materials used in the reactors [35]. We characterized the defects caused by $\mathrm{He}$ ion exposure in a correlative AFM-HIM experiment. Amorphous silicon bubbles are created on a crystalline silicon substrate through point exposition with the HIM at $25 \mathrm{kV}$ and $14 \mathrm{pA}$ using doses between $4.2 \times 10^{8}$ and $4.2 \times 10^{9} \mathrm{He}$ ions (Figure 3 ). He ions penetrate deep into the silicon and lead to the formation of microscale and nanoscale bubbles, which coalesce and ultimately result in the formation of a large silicon bubble in the amorphized silicon.
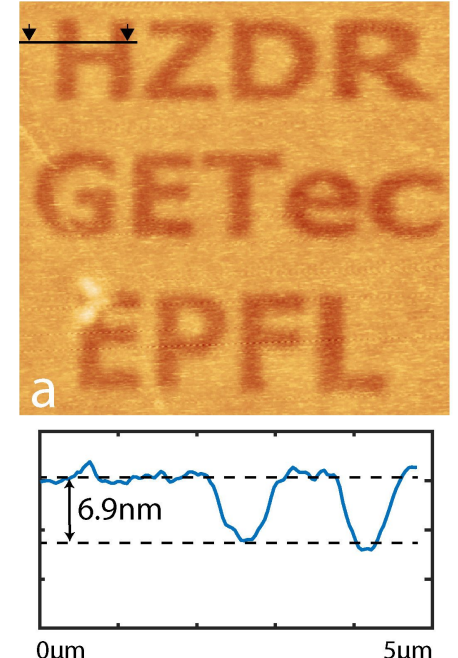

$0 \mu \mathrm{m}$
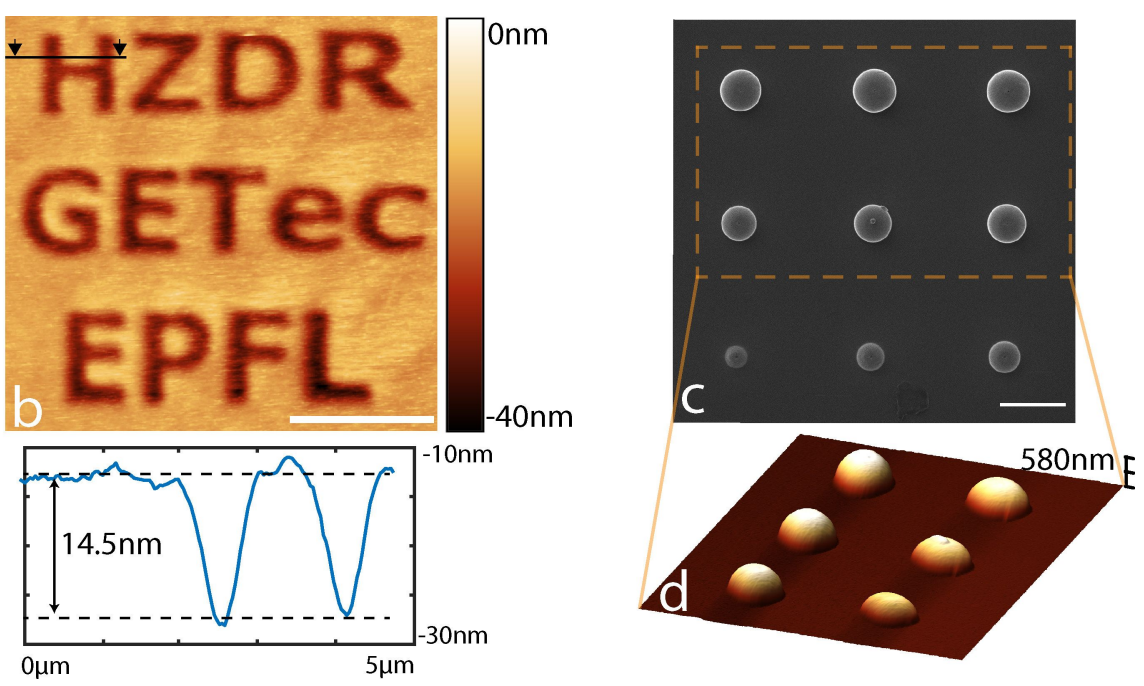

Figure 3: AFM height images of poly (methyl methacrylate) after exposure to a) $1 \times 10^{13}$ and b) $3 \times 10^{13} \mathrm{He}$ ion $\mathrm{cm}^{-2}$. The images were taken in contact mode, scale bar $4 \mu \mathrm{m}$. c) Silicon bubbles imaged with HIM (scale bar $1 \mu \mathrm{m}$ ) and d) AFM (off-resonance tapping mode). 
The resulting $3 \times 3$ bubble grid is imaged with HIM (Figure 3c) and AFM (Figure 3d) to reveal the height and volume of the features.

\section{Discussion}

For the successful integration of two different microscopy techniques, they should be both complementary and compatible. The techniques should, on the one hand, be sufficiently different so that the combination creates real added value. On the other hand, the application space of the techniques should have sufficient overlap so that a meaningful correlation can be established in space and time. One requirement for compatibility is that the AFM can operate in an ultra-high vacuum (UHV) environment, a prerequisite for the HIM. This requirement puts additional restrictions on the AFM. In our AFM design, we accounted for this already in the mechanical design (avoidance of trapped air pockets, no lubricants, UHV-compatible motors) as well as in the assembly by using, wherever possible, Kapton flex-PCBs or scarcely outgassing Teflon-coated wires. It is also critical that the AFM, the coarse stage, and the mounting elements are thoroughly cleaned before assembly and that assembly and storage are carried out in a clean environment. Disassembly and thorough cleaning of all the components prior to integration into the HIM was inadequately performed, which resulted in an inferior chamber vacuum pressure of $6.12 \times 10^{-7}$ mbar after overnight pumping, although normal HIM operation remained possible for our experiments. We strongly believe that if cleaning and storing recommendations are followed, the system vacuum would drastically improve, reaching more than adequate vacuum levels. It should also be noted that our AFM system is not compatible with baking the system at high temperatures above $100{ }^{\circ} \mathrm{C}$ since this would result in irreversible damage to the piezo actuators.

Another requirement for compatibility is that the two techniques can use samples prepared in the same way. For AFM and HIM, this is particularly advantageous since both can image non-conductive samples at very high resolution without charging. This is essential for correlative mechanical property and HIM surface imaging, and it is a clear advantage of AFM-HIM compared to AFM-SEM, where a conductive coating is often necessary for high-resolution SEM imaging.

The other aspect necessary for a useful integration of two techniques is that they are sufficiently complementary to each other to warrant the additional effort. While both AFM and HIM can yield images with very high resolution, the two techniques do have very different strengths. The HIM, for example, has a very good lateral resolution and a large depth of field, which makes it well suited for imaging high aspect ratio structures. The $Z$-resolution of the method, however, is less accurate, since the height of objects has to be reconstructed from two tilted images. AFM, on the other hand, has its highest resolution in the $Z$-direction, and profiles or volumes can be accurately extracted (see Figure 3d). The depth of field is, however, limited and the maximum slope of the sample that can be faithfully measured is dictated by the aspect ratio of the tip [36]. The true strength of the integrated setup is the combination of sample modification by the He ion beam and the multiparametric characterization of sample properties using AFM. In Figure 3, we showed a basic application where we characterized the effect of ion-beam radiation on the topography of the photoresist PMMA. Many more examples can be envisioned. The He ion beam is known to change the mechanical [37], electrical [38], and magnetic properties of materials [39]. AFM can be used to measure mechanical properties using contact resonance [40,41] or off-resonance tapping techniques [24] with very high resolution. Magnetic properties of nanostructures can be measured using magnetic force microscopy (MFM) [42], and a host of AFM techniques are available to measure electrical properties of samples (e.g., conductive AFM (cAFM) [43], scanning capacitance microscopy (SCM) [44], and spreading resistance microscopy (SSRM) [45]). While the implementation of these different imaging modes will require some additional modifications to our existing instrument, the path towards achieving such a truly multi-physics characterization and manipulation tool by combining advanced AFM with HIM can clearly be envisioned.

One aspect where HIM and AFM are, however, not well matched is in the image acquisition time. The relatively long time required for an AFM image (several minutes) has been a severe disadvantage when combining it with other electron or ion-beam microscopes. The same limitation exists for the combination of AFM and HIM. While much progress has been made towards increasing the imaging speed of AFM [46-50], most of this progress has been limited to imaging in liquid, due to the inherent bandwidth limitation of cantilevers when using them in dynamic mode in vacuum. Recent signs of progress in cantilever materials have shown the potential to increase the imaging speed of AFM also in ambient air or vacuum [51-53]. These approaches could also be implemented for the combined AFM-HIM instrument, thereby holding promise for interactive use of AFM and HIM at similar size- and time-scales.

\section{Conclusion}

We have demonstrated the integration of an atomic force microscope into a helium ion microscope. Correlative measurements of AFM topography with He ion imaging and modification demonstrate the feasibility of this integration. The complementarity of the two methods in terms of vertical and lateral resolution, nanoscale machining, and measurement of physical prop- 
erties of the sample will allow for a multi-physics investigation in many areas of materials science and technology, such as energy materials, magnetic nanostructures, and (bio-)composites.

\section{Experimental}

All AFM measurements were taken using silicon piezo-resistive self-sensing cantilevers (PRS-L100-F400-SCD-PCB SCLSensorTech Fabrication GMBH, Vienna, Austria) with a spring constant of ca. $100 \mathrm{~N} / \mathrm{m}$, and a footprint of $110 \times 48 \mu \mathrm{m}$. Imaging gains on the custom-made controller were adjusted as high as possible before significant oscillations were seen. AFM images were processed in the software Gwyddion [54]. Pixels were squared to account for $X-Y$ pixel size mismatch when necessary, the background was flattened and a conservative de-noising filter was applied. Finally, hysteresis correction was performed in MATLAB using closed-loop sensor data obtained prior to imaging on the AFM scan head.

For Figure 2, the AFM image shown was recorded at $300 \mathrm{mHz}$ line rate at a resolution of 1024 pixels and 512 lines and over a scan range of $30 \times 30 \mu \mathrm{m}$. The imaging mode used was offresonance tapping (ORT) at a tapping rate of $2 \mathrm{kHz}$ and a tapping amplitude of $600 \mathrm{~nm}$. For Figure $3 \mathrm{a}$ and Figure 3b, the images were taken in contact mode at $500 \mathrm{mHz}$ line rate and $1 \mathrm{~Hz}$ line rate respectively. Additionally, to the processing described in detail above, the two images were cropped and rotated to obtain the final images (the original images are $17.8 \times 17.8 \mu \mathrm{m}$ and $16.6 \times 16.6 \mu \mathrm{m}$, respectively, and each is $512 \times 512$ pixels). An additional 2-dimensional FFT filtering was applied to correct for the main mechanical vibrations in the two original images. The AFM image in Figure $3 \mathrm{~d}$ is obtained in ORT at $2 \mathrm{kHz}$ tapping rate, $600 \mathrm{~nm}$ amplitude and $200 \mathrm{mHz}$ line rate. The scan range is $9.7 \times 7.3 \mu \mathrm{m}$ and the image size is $512 \times 386$ pixels.

\section{Acknowledgements}

The authors acknowledge the support of Jeff Markakis for the mechanical design of the AFM scanner and Christian Schwalb for conceptual discussions.

\section{Funding}

This work was funded by the Swiss National Science Foundation (the Swiss National Science Foundation through grant 200021_182562), the European Union Eurostars Program (Eurostars E!8213), the H2020 IONS4SET project (H2020 Grant number: 688072), the Ion Beam Center of the Helmholtz Zentrum Dresden Rossendorf through GATE proposal 19001761 and the Austrian Research Promotion Agency FFG in the frame of the "Beyond Europe" initiative (Project AIM, No. 11056459).

\section{ORCID ${ }^{\circledR}$ iDs}

Gregor Hlawacek - https://orcid.org/0000-0001-7192-716X Stefan Hummel - https://orcid.org/0000-0002-4426-3609

Georg E. Fantner - https://orcid.org/0000-0001-5889-3022

\section{Preprint}

A non-peer-reviewed version of this article has been previously published as a preprint: https://arxiv.org/abs/2004.00536

\section{References}

1. Binnig, G.; Quate, C. F.; Gerber, C. Phys. Rev. Lett. 1986, 56, 930-933. doi:10.1103/physrevlett.56.930

2. Vázquez, L.; Bartolomé, A.; García, R.; Buendía, A.; Baró, A. M. Rev. Sci. Instrum. 1988, 59, 1286-1289. doi:10.1063/1.1139710

3. Ehrichs, E. E.; Smith, W. F.; de Lozanne, A. L. J. Vac. Sci. Technol., B: Microelectron. Nanometer Struct.-Process., M eas., Phenom. 1991, 9, 1380-1383. doi:10.1116/1.585201

4. Ermakov, A. V.; Garfunkel, E. L. Rev. Sci. Instrum. 1994, 65, 2853-2854. doi:10.1063/1.1144627

5. Stahl, U.; Yuan, C. W.; de Lozanne, A. L.; Tortonese, M. Appl. Phys. Lett. 1994, 65, 2878-2880. doi:10.1063/1.113030

6. Troyon, M.; Lei, H. N.; Wang, Z.; Shang, G. Microsc., Microanal., Microstruct. 1997, 8, 393-402. doi:10.1051/mmm:1997130

7. Fukushima, K.; Saya, D.; Kawakatsu, H. Jpn. J. Appl. Phys., Part 1 2000, 39, 3747-3749. doi:10.1143/jjap.39.3747

8. Mick, U.; Eichhorn, V.; Wortmann, T.; Diederichs, C.; Fatikow, S. Combined nanorobotic AFM/SEM system as novel toolbox for automated hybrid analysis and manipulation of nanoscale objects. In Proceedings of the 2010 IEEE International Conference on Robotics and Automation, 2010; pp 4088-4093. doi:10.1109/robot.2010.5509414

9. Angelov, T.; Ahmad, A.; Guliyev, E.; Reum, A.; Atanasov, I.; Ivanov, T.; Ishchuk, V.; Kaestner, M.; Krivoshapkina, Y.; Lenk, S.; Lenk, C.; Rangelow, I. W.; Holz, M.; Nikolov, N. J. Vac. Sci. Technol., B: Nanotechnol. Microelectron.: Mater., Process., Meas., Phenom. 2016, 34, 06KB01. doi:10.1116/1.4964290

10. Kreith, J.; Strunz, T.; Fantner, E. J.; Fantner, G. E.; Cordill, M. J. Rev. Sci. Instrum. 2017, 88, 053704. doi:10.1063/1.4983317

11. Rangelow, I. W.; Kaestner, M.; Ivanov, T.; Ahmad, A.; Lenk, S.; Lenk, C.; Guliyev, E.; Reum, A.; Hofmann, M.; Reuter, C.; Holz, M. J. Vac. Sci. Technol., B: Nanotechnol. Microelectron.: Mater., Process., Meas., Phenom. 2018, 36, 06J102. doi:10.1116/1.5048524

12. Ward, B. W.; Notte, J. A.; Economou, N. P. J. Vac. Sci. Technol., B: Microelectron. Nanometer Struct.-Process., M eas., Phenom. 2006, 24, 2871-2874. doi:10.1116/1.2357967

13. Hlawacek, G.; Veligura, V.; van Gastel, R.; Poelsema, B. J. Vac. Sci. Technol., B: Nanotechnol. Microelectron.: Mater., Process., Meas., Phenom. 2014, 32, 020801. doi:10.1116/1.4863676

14. Komuro, M.; Atoda, N.; Kawakatsu, H. J. Electrochem. Soc. 1979, 126, 483-490. doi:10.1149/1.2129067

15. Kalhor, N.; Alkemade, P. F. A. Resist assisted patterning. In NanoScience and Technology; Hlawacek, G.; Gölzhäuser, A., Eds.; Springer: Cham, Switzerland, 2016; pp 395-414. doi:10.1007/978-3-319-41990-9_16

16. Uruma, T.; Tsunemitsu, C.; Terao, K.; Nakazawa, K.; Satoh, N.; Yamamoto, H.; Iwata, F. AIP Adv. 2019, 9, 115011. doi:10.1063/1.5125163 
17. Yang, C.; Winkler, R.; Dukic, M.; Zhao, J.; Plank, H.; Fantner, G. E. ACS Appl. Mater. Interfaces 2017, 9, 24456-24461. doi:10.1021/acsami.7b07762

18. Leang, K. K.; Fleming, A. J. Asian J. Control 2009, 11, 144-153. doi:10.1002/asjc. 90

19. Tortonese, M.; Barrett, R. C.; Quate, C. F. Appl. Phys. Lett. 1993, 62, 834-836. doi:10.1063/1.108593

20. Fantner, G. E.; Burns, D. J.; Belcher, A. M.; Rangelow, I. W.; Youcef-Toumi, K. J. Dyn. Syst., Meas., Control 2009, 131, 061104. doi:10.1115/1.4000378

21. Fantner, G. E.; Schumann, W.; Barbero, R. J.; Deutschinger, A.; Todorov, V.; Gray, D. S.; Belcher, A. M.; Rangelow, I. W.; Youcef-Toumi, K. Nanotechnology 2009, 20, 434003. doi:10.1088/0957-4484/20/43/434003

22. Obraztsov, A. N.; Kopylov, P. G.; Loginov, B. A.; Dolganov, M. A.; Ismagilov, R. R.; Savenko, N. V. Rev. Sci. Instrum. 2010, 81, 013703. doi:10.1063/1.3280182

23. Nievergelt, A. P.; Brillard, C.; Eskandarian, H. A.; McKinney, J. D.; Fantner, G. E. Int. J. Mol. Sci. 2018, 19, 2984. doi:10.3390/ijms19102984

24. Rosa-Zeiser, A.; Weilandt, E.; Hild, S.; Marti, O. Meas. Sci. Technol. 1997, 8, 1333-1338. doi:10.1088/0957-0233/8/11/020

25. Nievergelt, A. P.; Erickson, B. W.; Hosseini, N.; Adams, J. D.; Fantner, G. E. Sci. Rep. 2015, 5, 11987. doi:10.1038/srep11987

26. de Pablo, P. J.; Colchero, J.; Gómez-Herrero, J.; Baró, A. M. Appl. Phys. Lett. 1998, 73, 3300-3302. doi:10.1063/1.122751

27. Alsteens, D.; Dupres, V.; Yunus, S.; Latgé, J.-P.; Heinisch, J. J.; Dufrêne, Y. F. Langmuir 2012, 28, 16738-16744. doi:10.1021/la303891j

28. Mertz, J.; Marti, O.; Mlynek, J. Appl. Phys. Lett. 1993, 62, 2344-2346. doi:10.1063/1.109413

29. Jaafar, M.; Martínez-Martín, D.; Cuenca, M.; Melcher, J.; Raman, A.; Gómez-Herrero, J. Beilstein J. Nanotechnol. 2012, 3, 336-344. doi:10.3762/bjnano.3.38

30. Xu, X.; Prüfer, T.; Wolf, D.; Engelmann, H.-J.; Bischoff, L.; Hübner, R.; Heinig, K.-H.; Möller, W.; Facsko, S.; von Borany, J.; Hlawacek, G. Beilstein J. Nanotechnol. 2018, 9, 2883-2892. doi:10.3762/bjnano.9.267

31. Calcagno, L.; Compagnini, G.; Foti, G. Nucl. Instrum. Methods Phys. Res., Sect. B 1992, 65, IN7-422. doi:10.1016/0168-583x(92)95077-5

32. Kallweit, R.; Baur, M.; Eichinger, P.; Strack, H. Nucl. Instrum. Methods Phys. Res., Sect. B 1991, 59-60, 1288-1291. doi:10.1016/0168-583x(91)95813-s

33. Wang, Y.; Boden, S. A.; Bagnall, D. M.; Rutt, H. N.; de Groot, C. H. Nanotechnology 2012, 23, 395302. doi:10.1088/0957-4484/23/39/395302

34. Livengood, R.; Tan, S.; Greenzweig, Y.; Notte, J.; McVey, S. J. Vac. Sci. Technol., B: Microelectron. Nanometer Struct.-Process., M eas., Phenom. 2009, 27, 3244-3249. doi:10.1116/1.3237101

35. Guo, Q.; Landau, P.; Hosemann, P.; Wang, Y.; Greer, J. R. Small 2013, 9, 691-696. doi:10.1002/smll.201201614

36. Akamine, S.; Barrett, R. C.; Quate, C. F. Appl. Phys. Lett. 1990, 57, 316-318. doi:10.1063/1.103677

37. Bergner, F.; Hlawacek, G.; Heintze, C. J. Nucl. Mater. 2018, 505, 267-275. doi:10.1016/j.jnucmat.2017.07.054
38. Fox, D. S.; Zhou, Y.; Maguire, P.; O’Neill, A.; Ó’Coileáin, C.; Gatensby, R.; Glushenkov, A. M.; Tao, T.; Duesberg, G. S.; Shvets, I. V.; Abid, M.; Abid, M.; Wu, H.-C.; Chen, Y.; Coleman, J. N.; Donegan, J. F.; Zhang, H. Nano Lett. 2015, 15, 5307-5313. doi:10.1021/acs.nanolett.5b01673

39. Franken, J. H.; Hoeijmakers, M.; Lavrijsen, R.; Kohlhepp, J. T.; Swagten, H. J. M.; Koopmans, B.; van Veldhoven, E.; Maas, D. J. J. Appl. Phys. 2011, 109, 07D504. doi:10.1063/1.3549589

40. Rabe, U.; Arnold, W. Appl. Phys. Lett. 1994, 64, 1493-1495. doi:10.1063/1.111869

41. Yamanaka, K.; Nakano, S. Jpn. J. Appl. Phys., Part 1 1996, 35 , 3787-3792. doi:10.1143/jjap.35.3787

42. Martin, Y.; Wickramasinghe, H. K. Appl. Phys. Lett. 1987, 50, 1455-1457. doi:10.1063/1.97800

43. Murrell, M. P.; Welland, M. E.; O'Shea, S. J.; Wong, T. M. H.; Barnes, J. R.; McKinnon, A. W.; Heyns, M.; Verhaverbeke, S. Appl. Phys. Lett. 1993, 62, 786-788. doi:10.1063/1.108579

44. Matey, J. R.; Blanc, J. J. Appl. Phys. 1985, 57, 1437-1444. doi:10.1063/1.334506

45. Eyben, P.; Vandervorst, W.; Alvarez, D.; Xu, M.; Fouchier, M. Probing semiconductor technology and devices with scanning spreading resistance microscopy. Scanning Probe Microscopy; Springer: New York, NY, USA, 2007; Vol. 2, pp 31-87. doi:10.1007/978-0-387-28668-6_3

46. Kodera, N.; Yamamoto, D.; Ishikawa, R.; Ando, T. Nature 2010, 468, 72-76. doi:10.1038/nature09450

47. Fantner, G. E.; Schitter, G.; Kindt, J. H.; Ivanov, T.; Ivanova, K.; Patel, R.; Holten-Andersen, N.; Adams, J.; Thurner, P. J.; Rangelow, I. W.; Hansma, P. K. Ultramicroscopy 2006, 106, 881-887. doi:10.1016/j.ultramic.2006.01.015

48. Hansma, P. K.; Schitter, G.; Fantner, G. E.; Prater, C. Science 2006, 314, 601-602. doi:10.1126/science.1133497

49. Ando, T.; Kodera, N.; Takai, E.; Maruyama, D.; Saito, K.; Toda, A. Proc. Natl. Acad. Sci. U. S. A. 2001, 98, 12468-12472. doi:10.1073/pnas.211400898

50. Humphris, A. D. L.; Miles, M. J.; Hobbs, J. K. Appl. Phys. Lett. 2005, 86, 034106. doi:10.1063/1.1855407

51. Adams, J. D.; Erickson, B. W.; Grossenbacher, J.; Brugger, J.; Nievergelt, A.; Fantner, G. E. Nat. Nanotechnol. 2016, 11, 147-151. doi:10.1038/nnano.2015.254

52. Alsharif, N.; Burkatovsky, A.; Lissandrello, C.; Jones, K. M.; White, A. E.; Brown, K. A. Small 2018, 14, 1800162. doi:10.1002/smll.201800162

53. Hosseini, N.; Neuenschwander, M.; Peric, O.; Andany, S. H.; Adams, J. D.; Fantner, G. E. Beilstein J. Nanotechnol. 2019, 10, 2357-2363. doi:10.3762/bjnano.10.226

54. Nečas, D.; Klapetek, P. Cent. Eur. J. Phys. 2012, 10, 181-188. doi:10.2478/s11534-011-0096-2 


\section{License and Terms}

This is an Open Access article under the terms of the Creative Commons Attribution License (https://creativecommons.org/licenses/by/4.0). Please note that the reuse, redistribution and reproduction in particular requires that the authors and source are credited.

The license is subject to the Beilstein Journal of Nanotechnology terms and conditions: (https://www.beilstein-journals.org/bjnano)

The definitive version of this article is the electronic one which can be found at:

https://doi.org/10.3762/bjnano.11.111 